\title{
Protective Effects of Biochanin A against Methotrexate-Induced Acute Liver Injury in Rats
}

\author{
Erdem Kamil Ozer ${ }^{1 *}$, Mustafa Tugrul Goktas ${ }^{2}$, Aysun Toker $^{3}$, Ceyhan Ugurluoglu ${ }^{4}$, Hulagu Bariskaner ${ }^{1}$ \\ ${ }^{1}$ Department of Pharmacology, Faculty of Medicine, Selcuk University, Konya, TURKEY. \\ ${ }^{2}$ Department of Pharmacology, Faculty of Medicine, Yildirim Beyazit University, Ankara, TURKEY. \\ ${ }^{3}$ Department of Biochemistry, Faculty of Medicine, Necmettin Erbakan University, Konya, TURKEY. \\ ${ }^{4}$ Department of Pathology, Faculty of Medicine, Selcuk University, Konya, TURKEY.
}

\begin{abstract}
Background: Methotrexate (MTX) is one of the most commonly used chemotherapeutic agents in the treatment of cancer patients, however its therapeutic use is limited due to dose dependent hepatotoxicity caused by oxidative damage. Biochanin A (BCA), a naturally occurring dietary isoflavone, has strong cytoprotective, anti-inflammatory, and antioxidant properties. Although protective actions of BCA against various chemicalinduced hepatotoxicity including that of carbon tetrachloride and arsenic, none of the studies was made on MTX-induced acute liver injury. Methods: Wistar rats were separated into four groups; Control, MTX, Control + BCA, MTX+BCA. BCA (50 mg/kg/day) or vehicle (dimethyl sulfoxide; $1 \mathrm{~mL} / \mathrm{kg} / \mathrm{day}$ ) with the same volume was intraperitoneally injected for 5 days. At sixth day, a single dose of MTX $(20 \mathrm{mg} / \mathrm{kg})$ was injected to rats. Twenty-four $\mathrm{h}$ after the MTX administration, rats were sacrificed and then blood and liver samples were obtained for biochemical and histopathologic analyses.Results: MTX increased the serum AST, ALT, and LDH levels. Furthermore, malondialdehyde (an indicator of oxidative injury) and myeloperoxidase (an indicator of neutrophil infiltration) levels increased, while total glutathione levels (an indicator of antioxidant status) decreased in liver. MTX-induced hepatotoxicity was also observed histopathologically. BCA substantially improved these alterations induced by MTX administration. Conclusion: These results indicate that BCA may be useful in preventing the MTX-induced acute liver injury due to its antioxidant and anti-inflammatory properties.
\end{abstract}

Key words: Methotrexate, Hepatotoxicity, Biochanin A, Protective effects

\section{INTRODUCTION}

Methotrexate (MTX), a folic acid antagonist, has dose-dependent cytotoxic and immunosuppressive effects that has been used for many years to treat a wide range of disease including different types of inflammatory diseases and cancer., ${ }^{1,2}$ Despite current widespread use of MTX, hepatotoxicity, one of the serious complications of MTX, limits its use and leads to malabsorption and diarrhea. ${ }^{3,45}$ the exact underlying pathophysiological mechanism of MTX-induced hepatotoxicity is not well known. However, several studies have suggested that MTX triggers neutrophil infiltration, lipid peroxidation, and excessive release of free radicals.
Free radicals-induced oxidative stress can lead to apoptosis, cell death, and tissue damage. ${ }^{6,78}$ Therefore, it is believed that administration of antioxidant may inhibit the MTX-induced liver damage.

Biochanin A (5, 7-Dihydroxy-4'-methoxyisoflavone, BCA) is a member of a natural organic compound classified under the phytochemicals known as isoflavonoids. BCA is predominantly found in red clover, soy, peanuts, and alfalfa sprouts. ${ }^{9,10}$ Recent studies have found that BCA possesses strong anti-oxidant, anti-inflammatory, and cytoprotective effects. ${ }^{11,12,13,14}$ However, there
Submission Date: 05-09-2017; Revision Date: 13-10-2017; Accepted Date: 27-12-2017.

DOI: 10.5530/ijper.51.4s.107 Correspondence:

Dr. Erdem Kamil Ozer, Selcuk University, Faculty of Medicine, Department of Pharmacology Konya-42250, TURKEY.

Phone: +90 3322243839

Fax: +90 3322412184

E-mail: drekfarma@hotmail. com

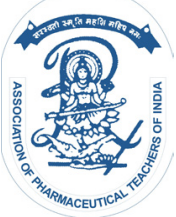

www.ijper.org 
are no detailed studies on potential beneficial effects of BCA against MTX-induced hepatotoxicity.

In the light of above information, it was aimed to examine whether pre administration of BCA can protect against MTX-induced liver damage.

\section{MATERIALS AND METHODS}

\section{Animals and experimental protocols}

Twenty-eight Wistar albino female rats aged 75-90 days and weighing between 250-300 g were used for this study (Experimental Research Center of Selcuk University, Konya, Turkey). They were maintained in an air controlled room with a 12:12 h light/dark illumination sequence at $50 \pm 10 \%$ humidity and $21{ }^{\circ} \mathrm{C}$ temperature. Animals had ad libitum access to commercial laboratory rodent pellet diet and tap water (drinking bottle). During the experiments animals to be randomly distributed in different cages following the each procedure and all animals were housed in the same animal room. This study was conducted in accordance with the standards for the care and use of animal subjects, as mentioned in the Guide for the Care and Use of Laboratory Animals (2011, published by National Academies Press) and Animal Ethics Committee of Selcuk University approved the protocol (Approval Number: 2015/57).

Animals were randomly separated into four subgroups as follows: Control, MTX, MTX+BCA, Control+BCA. The animals in the MTX+BCA and Control+BCA groups were intraperitoneally injected with BCA (50 $\mathrm{mg} / \mathrm{kg} /$ day) for 5 consecutive days. At the same time, rats in the Control and MTX groups were intraperitoneally injected with dimethyl sulfoxide (DMSO) as the solvent and vehicle of BCA at the same volume. After the BCA and vehicle administrations, MTX was given to rats in the MTX and MTX+BCA groups at a single dose of $20 \mathrm{mg} / \mathrm{kg}$ and physiological saline was intraperitoneally injected to rats in the Control and Control+ BCA groups at the same volume. ${ }^{6,11,12,15}$ Variations in body weight and behavioural changes were monitored during the period of experiment. Blood and tissue samples were collected $24 \mathrm{~h}$ after the last injection. Rats were fasted overnight one day before the sample collection. Animals were fasted overnight and intraperitoneally injected with sodium pentobarbital $(60 \mathrm{mg} / \mathrm{kg})$ to induce general anesthesia. Approximately $1 \mathrm{~mL}$ blood sample was collected into the Eppendorf tubes via cardiac puncture, abdominal cavity was then opened and liver tissue was carefully removed and weighed. Following this procedure rats were immediately euthanized by cervical dislocation. The piece of liver tissue was transferred into the Eppendorf tubes and stored at
$-80{ }^{\circ} \mathrm{C}$ until myeloperoxidase (MPO), malondialdehyde (MDA), and total glutathione measurements. After waiting 15-20 min blood specimens were centrifuged for $5 \mathrm{~min}$ at $4000 \mathrm{xg}$ and obtained aliquots of serum were frozen and stored at $-80^{\circ} \mathrm{C}$ for several biochemical assays. Additionally, rest of liver tissue was harvested and fixed in the solution of $10 \%$ neutral buffered formalin for histopathological analysis.

\section{Biochemical analyses}

The frozen aliquots of serum were thawed and then mixed. Hepatic function markers, aspartate aminotransferase (AST) and alanine aminotransferase (ALT), and as well as lactate dehydrogenase (LDH; a nonspecific indicator for tissue injury) were calculated with the standard auto analyzer methods on the Abbott Architect c16000 system by using the commercial reagents in accordance with the producer's protocols (Abbott Laboratories, Illinois, USA).

On the day of biochemical analyses in the liver tissues, tissue samples were homogenized in cold potassium phosphate-buffered solution $(\mathrm{pH}=7.4,50 \mathrm{mM}$, and $10 \%$ weight/volume) on an ice cube with a homogenizer (IKA T10 basic Ultra-Turrax Homogenizer). The obtained homogenate was centrifuged at $10000 \times g$ for $20 \mathrm{~min}$ and then clear supernatant was separated for biochemical measurements. MDA, an end product of lipid peroxidation, is a useful marker for oxidative stress-induced organ damage. To evaluate the antioxidant capacity of liver samples, total glutathione (reduced and oxidized forms) levels were assayed. MPO is an early biomarker of inflammation and neutrophil activation. The MDA and total glutathione levels, as well as MPO activity in liver tissues were determined with the commercial kits in accordance with the manufacturer protocol.

\section{Histopathological Examination}

The formaldehyde-fixed tissues were embedded into paraffin and cut in $5-\mu \mathrm{m}$ thick sections. The obtained sections were placed on slides and stained by hematoxylin and eosin. Microscopic organ damage scoring was performed by experienced pathologist. The pathologist was unaware of the treatments and evaluated tissue sections with a conventional light microscope (Carl Zeiss Axio Lab.A1) and photographed them with the camera of Axiocam MRc5 microscope. Severity of liver damage was semiquantitatively scored by the blinded pathologist as 0 (absent), 1 (slight), 2 (moderate), and 3 (severe) for each criteria including congestion/hemorrhage, inflammation, necrosis/degeneration, and sinusoidal dilatation. ${ }^{16}$ 


\section{Drugs and chemicals}

MTX was purchased from Kocak Pharmaceuticals (Istanbul, Turkey). BCA (Cat. No. D2016), sodium pentobarbital (P-010), total glutathione assay kit (CS0260), lipid peroxidation (MDA) assay kit (MAK085), and MPO Colorimetric Activity Assay Kit (MAK068) were provided by Sigma Aldrich (St Louis, MO, USA). Formaldehyde, paraffin, hematoxylin, and eosin were obtained from Merck Millipore (Billerica, MA, USA).

\section{Statistical analyses}

GraphPad Prism 5 software (San Diego, California, USA) was used for analysis of data. All data were expressed as mean \pm standard deviation (mean $\pm \mathrm{SD}$ ) and analyzed by using one-way analysis of variance (ANOVA) with Newman-Keuls multiple comparison post-test. A value of $p<0.05$ was considered as statistically significant. $n=7$ for each group. *Denotes significant difference between Controls vs. other groups. \#denotes significant difference between MTX vs. MTX+BCA group $\left(^{*}, \#\right.$, $\mathrm{p}<0.05 ; * *, \# \#, \mathrm{p}<0.01 ; * * *, \# \# \#, \mathrm{p}<0.001)$.

\section{RESULTS}

The body weight and also weight of liver were shown in Table 1. MTX did not effect the weights of body and liver. MTX administered rats showed clinical signs such as dullness, hunched posture, anorexia, and lethargy. Besides, there was no death during the experimental period.

\section{Effects of BCA on biochemical measurements}

Increases of the serum ALT and LDH levels caused by MTX administration were completely prevented by
BCA. Additionally, BCA improved the MTX-caused elevation in the serum AST levels but could not totally blocked Table 2. As shown in Table 3, liver MDA and MPO levels were elevated in the MTX group when compared with Control group $(p<0.001$ and $p<0.01)$. Pretreatment with BCA completely blocked the MTXcaused elevations of MDA and MPO levels in liver tissues. Moreover, MTX led to a significant reduction in total glutathione levels of the liver tissues when compared with Control group $(\phi<0.01)$. BCA also improved the MTX-induced reduction of total glutathione levels in the liver but could not totally block.

\section{Histopathological changes in liver by BCA}

The structures of liver samples were normal in the Control Figure-1A and Control+BCA Figure 1D groups. However, MTX caused substantial histopathological changes in the liver samples including mild to moderate sinusoidal and portal hemorrhage, mild inflammatory mononuclear cell infiltration in periportal area and parenchyma, dilatation of sinusoids, centrilobular and periportal degeneration and necrosis Figure 1B. When the rats were treated with $\mathrm{BCA}$ the liver Figure $1 \mathrm{C}$ were totally prevented from the histopathological damage caused by MTX. Furthermore, beneficial effects of BCA on MTX-caused organ injury were also shown in Table 4 with histopathological damage scoring.

\section{DISCUSSION}

MTX is now used to treat a wide variety of disorders. Approaches for reducing the MTX treatment-induced hepatotoxicity complications are valuable for success of the treatment and improving the life quality of the

\begin{tabular}{|c|c|c|c|c|}
\hline \multirow{2}{*}{} & \multicolumn{3}{|c|}{ Table 1: General outcome parameters of the rats. } \\
\cline { 2 - 3 } & Initial & Final & Liver weight (g) & Liver-Body weight Ratio (\%) \\
\hline Control & $272 \pm 13$ & $276 \pm 16$ & $22.71 \pm 2.14$ & $8.25 \pm 0.93$ \\
\hline MTX & $267 \pm 15$ & $276 \pm 14$ & $22.02 \pm 2.16$ & $7.99 \pm 1.01$ \\
\hline MTX+BCA & $264 \pm 15$ & $278 \pm 18$ & $23.86 \pm 1.95$ & $8.64 \pm 1.11$ \\
\hline Control+BCA & $277 \pm 8$ & $297 \pm 15$ & $23.14 \pm 1.35$ & $7.82 \pm 0.78$ \\
\hline
\end{tabular}

\begin{tabular}{|c|c|c|c|}
\hline \multicolumn{4}{|c|}{ Table 2: Serum biochemical markers. } \\
\hline & AST (IU/L) & ALT (IU/L) & LDH (IU/L) \\
\hline Control & $76 \pm 27$ & $34 \pm 12$ & $232 \pm 68$ \\
\hline MTX & $244 \pm 142^{* *}$ & $99 \pm 67^{*}$ & $463 \pm 208^{*}$ \\
\hline MTX+BCA & $165 \pm 71^{*}, \#$ & $57 \pm 28^{\#}$ & $291 \pm 193^{\#}$ \\
\hline Control+BCA & $69 \pm 23$ & $30 \pm 5$ & $222 \pm 69$ \\
\hline
\end{tabular}

\begin{tabular}{|c|c|c|c|}
\hline \multicolumn{4}{|c|}{ Table 3: Tissue oxidative stress parameters. } \\
\hline & $\begin{array}{c}\text { Liver MDA } \\
\text { (nmol/mg) }\end{array}$ & $\begin{array}{c}\text { Liver glutathione } \\
\text { (nmol/mg) }\end{array}$ & $\begin{array}{c}\text { Liver MPO } \\
\text { (mU/mL) }\end{array}$ \\
\hline Control & $6.83 \pm 0.47$ & $189 \pm 40$ & $57 \pm 12$ \\
\hline MTX & $9.98 \pm 2.29^{* * *}$ & $74 \pm 42^{* *}$ & $92 \pm 37^{* *}$ \\
\hline MTX+BCA & $6.73 \pm 0.85^{\ldots}$ & $137 \pm 31^{*}$ & $40 \pm 17^{\#}$ \\
\hline Control+BCA & $6.66 \pm 0.89$ & $185 \pm 52$ & $38 \pm 13$ \\
\hline
\end{tabular}




\begin{tabular}{|c|c|c|c|c|}
\hline \multicolumn{5}{|c|}{ Table 4: Histopathological organ damage scores. } \\
\hline & $\begin{array}{c}\text { congestion/ } \\
\text { hemorrhage }\end{array}$ & Inflammation & $\begin{array}{c}\text { necrosis/ } \\
\text { degeneration }\end{array}$ & sinusoidal dilatation \\
\hline Control & $0.14 \pm 0.38$ & $0.00 \pm 0.00$ & $0.00 \pm 0.00$ & $0.00 \pm 0.00$ \\
\hline MTX & $1.14 \pm 0.38^{* * *}$ & $0.86 \pm 0.69^{* *}$ & $0.71 \pm 0.49^{* *}$ & $0.57 \pm 0.53^{*}$ \\
\hline MTX+BCA & $0.29 \pm 0.49^{\# \#}$ & $0.14 \pm 0.38^{\#}$ & $0.14 \pm 0.38^{\#}$ & $0.14 \pm 0.38^{\#}$ \\
\hline Control+BCA & $0.14 \pm 0.38$ & $0.00 \pm 0.00$ & $0.00 \pm 0.00$ & $0.00 \pm 0.00$ \\
\hline
\end{tabular}
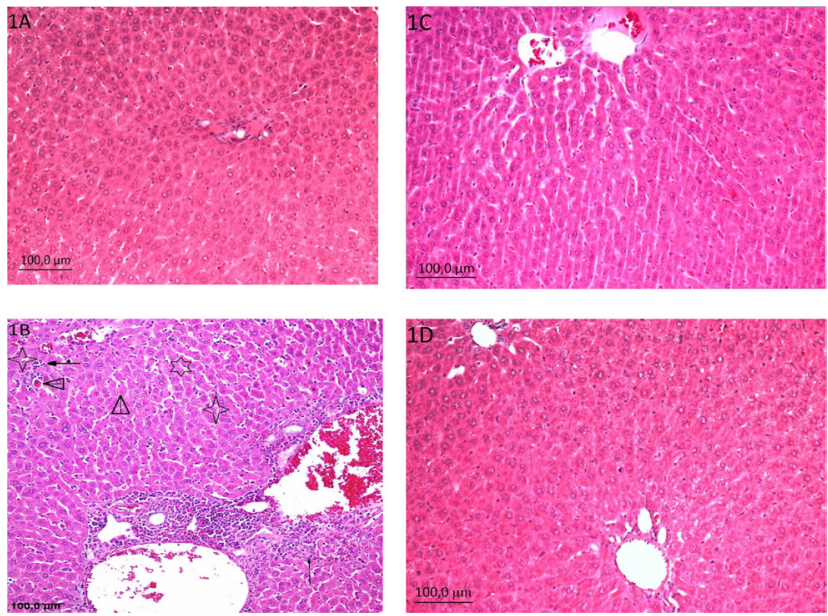

Figure 1: Effects of BCA on MTX-induced histopathological changes in the liver samples. The photographs show the representative histopathological liver sections that were obtained from the rats in Control (1A), MTX (1B), MTX+BCA (1C), and Control+BCA (1D) groups. The liver tissues show normal histopathological structures in the Control group.

There are also normal liver in the Control+BCA group.

Figure-1B shows the liver tissues in the MTX group with mild

to moderate sinusoidal portal hemorrhage (4-point satar), mild inflammatory mononuclear cell infiltration in the parenchyma and periportal area (arrow), dilatation of sinusoids (6-point star), centrilobular and periportal degeneration and necrosis (arrowhead). Figure-1C; normal liver architecture in MTX+BCA group.

patients. ${ }^{4,17}$ Liver is the main organ for the metabolism of MTX, therefore elevated free radical generation in the hepatic tissues due to use of MTX is associated with the hepatotoxic effects. ${ }^{6,7}$ Free radicals mainly target the cell membrane lipids that can lead to cellular damage via peroxidation of lipids. ${ }^{18}$ The liver is highly vulnerable to oxidative stress due to its high lipid ingredient. ${ }^{19,20}$ The deleterious effects of oxidative damage caused by MTX could be attributed to lipid peroxidation. . $^{6,7,8,21}$ MDA levels, an end product of lipid peroxidation chain and a useful marker for lipid peroxidation, are known to be increased in response to oxidative stress status. ${ }^{16,22}$ The result of increased liver MDA levels in the present study suggests the presence of increased lipid peroxidation induced by MTX. MPO, a heme-containing enzyme and mainly located in neutrophils, is released in high amounts in the process of inflammation. MPO generates hypochlorous acid utilizing the hydrogen peroxide and chloride. Hypochlorous acid is a strong oxidizing agent that produces the free radicals during the oxidation of hydrazines. ${ }^{6,23,24}$ Glutathione, a small tripeptide and the major free thiol in most mammalian cells, plays a role in many important biological processes including removal of hydroperoxides, detoxification of xenobiotic, scavenging free radicals, DNA synthesis, and maintenance of the oxidation state of protein sulfhydryls. ${ }^{16,25,26}$ AST and ALT are present in high concentration in the hepatocytes, however loss of hepatocyte membrane integrity due to lipid peroxidation and free radicals leads to release of AST, ALT, as well as LDH into the bloodstream.

In our study we showed that administration of MTX caused oxidative tissue injury as determined by increased lipid peroxidation, excessive free radical production via activated neutrophils, whereas depletion of antioxidant defense system in the liver in accordance with the literature. Furthermore, we also observed histopathological deleterious effects of MTX administration in the liver. Thus, agents like BCA that have strong antioxidative and anti-inflammatory properties may inhibit the MTXinduced liver injury.

BCA has shown potent antioxidant, anti-inflammatory, and cytoprotective effects on various experimental studies such as lipopolysaccharide/D-galactosamine-induced acute liver injury, ${ }^{13}$ ethanol-induced gastric mucosal ulceration, ${ }^{12}$ and cerebral ischemia/reperfusion injury. ${ }^{14}$ Arsenic and carbon tetrachloride are well-known hepatotoxins that lead to hepatocyte degeneration, necrosis, and inflammation. Jalaludeen et al. showed that BCA ameliorated the arsenic-induced hepatotoxicity in rats. ${ }^{27}$ Furthermore, it was shown that BCA has protective effects against hepatotoxicity induced by carbon tetrachloride.$^{11}$ However, to the best of our knowledge, there is no study examining the possible protective effects of BCA on MTX-induced acute liver injury.

In our study, it was found that BCA prevented the hepatotoxicity induced by MTX administration in rats. This could be due to suppression of the lipid peroxidation, free radical production, and neutrophil activity, whereas boost of the antioxidant capacity and hepatocyte membrane integrity and function. 


\section{CONCLUSION}

Our study reports that BCA has hepatoprotective effects against acute liver injury caused by MTX by reducing oxidative stress and inflammatory process. According to these results, pretreatment with BCA may provide a novel therapeutic approach for prevention of MTXinduced hepatotoxicity. Additional investigations are also required to show the clinical implication of BCA in patients receiving MTX.

\section{ACKNOWLEDGEMENT}

The present study did not receive any specific grant from any funding agency in the commercial, public, or not-for-profit sectors.

\section{CONFLICT OF INTEREST}

The authors declare that there is no conflict of interest.

\section{ABBREVIATIONS USED}

MTX: Methotrexate; BCA: Biochanin A; DMSO: dimethyl sulfoxide; MPO: Myeloperoxidase; MDA: malondialdehyde; AST: Aspartat aminotransferase; ALT: Alanine aminotransferase; LDH: Lactate dehydrogenase; ANOVA: One-way analysis of variance.

\section{REFERENCES}

1. Padmanabhan S, Tripathi DN, Vikram A, Ramarao $P$ and Jena GB. Methotrexate-induced cytotoxicity and genotoxicity in germ cells of mice: intervention of folic and folinic acid. Mutat Res. 2009; 673(1): 43-52.

2. Wolfe $F$ and Michaud K. Lymphoma in rheumatoid arthritis: the effect of methotrexate and anti-tumor necrosis factor therapy in 18,572 patients. Arthritis Rheum. 2004; 50(6): 1740-51.

3. de Araujo AA, Borba PB, de Souza FH, et al. In a methotrexate-induced model of intestinal mucositis, olmesartan reduced inflammation and induced enteropathy characterized by severe diarrhea, weight loss, and reduced sucrose activity. Biol Pharm Bull. 2015; 38: 746-52.

4. Howard SC, McCormick J, Pui CH, Buddington RK and Harvey RD. Preventing and Managing Toxicities of High-Dose Methotrexate. Oncologist. 2016; 21: 1471-82.

5. Valentino PL, Church PC, Shah PS, et al. Hepatotoxicity caused by methotrexate therapy in children with inflammatory bowel disease: a systematic review and meta-analysis. Inflamm Bowel Dis. 2014; 20: 47-59.

6. Cetinkaya A, Bulbuloglu E, Kurutas EB and Kantarceken B. N-acetylcysteine ameliorates methotrexate-induced oxidative liver damage in rats. Med Sci Monit. 2006; 12: BR274-8.

7. Mahmoud AM, Hussein OE, Hozayen WG and Abd El-Twab SM. Methotrexate hepatotoxicity is associated with oxidative stress, and down-regulation of
PPARgamma and Nrf2: Protective effect of 18beta-Glycyrrhetinic acid. Chem Biol Interact. 2017; 270: 59-72.

8. Moghadam AR, Tutunchi S, Namvaran-Abbas-Abad A, et al. Preadministration of turmeric prevents methotrexate-induced liver toxicity and oxidative stress. BMC Complement Altern Med. 2015; 15: 246.

9. Harini R, Ezhumalai $M$ and Pugalendi KV. Antihyperglycemic effect of biochanin $\mathrm{A}$, a soy isoflavone, on streptozotocin-diabetic rats. Eur $J$ Pharmacol. 2012; 676: 89-94.

10. Lapcik O, Vitkova M, Klejdus B, Al-Maharik $\mathrm{N}$ and Adlercreutz $\mathrm{H}$. Immunoassay for biochanin A. J Immunol Methods. 2004; 294: 155-63.

11. Breikaa RM, Algandaby MM, El-Demerdash E and Abdel-Naim AB. Biochanin A protects against acute carbon tetrachloride-induced hepatotoxicity in rats. Biosci Biotechnol Biochem. 2013; 77: 909-16.

12. Hajrezaie $\mathrm{M}$, Salehen $\mathrm{N}$, Karimian $\mathrm{H}$, et al. Biochanin a gastroprotective effects in ethanol-induced gastric mucosal ulceration in rats. PLoS One. 2015; 10: e0121529.

13. Liu X, Wang T, Liu X, et al. Biochanin A protects lipopolysaccharide/Dgalactosamine-induced acute liver injury in mice by activating the Nrf2 pathway and inhibiting NLRP3 inflammasome activation. Int Immunopharmacol. 2016; 38: 324-31.

14. Wang W, Tang L, Li Y and Wang Y. Biochanin A protects against focal cerebral ischemia/reperfusion in rats via inhibition of p38-mediated inflammatory responses. J Neurol Sci. 2015; 348: 121-5.

15. Moon YJ, Sagawa K, Frederick K, Zhang S and Morris ME. Pharmacokinetics and bioavailability of the isoflavone biochanin A in rats. AAPS J. 2006; 8: E433-42.

16. Ozer EK, Goktas MT, Toker A, Bariskaner H, Ugurluoglu $C$ and Iskit $A B$. Effects of Carvacrol on Survival, Mesenteric Blood Flow, Aortic Function and Multiple Organ Injury in a Murine Model of Polymicrobial Sepsis. Inflammation. 2017.

17. Tilling L, Townsend $S$ and David J. Methotrexate and hepatic toxicity in rheumatoid arthritis and psoriatic arthritis. Clin Drug Investig. 2006; 26: 55-62.

18. Pamplona R. Membrane phospholipids, lipoxidative damage and molecular integrity: a causal role in aging and longevity. Biochim Biophys Acta. 2008; 1777: 1249-62.

19. Cichoz-Lach $\mathrm{H}$ and Michalak $\mathrm{A}$. Oxidative stress as a crucial factor in liver diseases. World J Gastroenterol. 2014; 20: 8082-91.

20. de Andrade KQ, Moura FA, dos Santos JM, de Araujo OR, de Farias Santos JC and Goulart MO. Oxidative Stress and Inflammation in Hepatic Diseases: Therapeutic Possibilities of N-Acetylcysteine. Int J Mol Sci. 2015; 16: 30269308.

21. Bath RK, Brar NK, Forouhar FA and Wu GY. A review of methotrexateassociated hepatotoxicity. J Dig Dis. 2014; 15: 517-24.

22. Ayala A, Munoz MF and Arguelles S. Lipid peroxidation: production, metabolism, and signaling mechanisms of malondialdehyde and 4-hydroxy2-nonenal. Oxid Med Cell Longev. 2014; 2014: 360438.

23. Loria V, Dato I, Graziani F and Biasucci LM. Myeloperoxidase: a new biomarker of inflammation in ischemic heart disease and acute coronary syndromes. Mediators Inflamm. 2008; 2008: 135625.

24. Shaeib F, Khan SN, Thakur M, et al. The Impact of Myeloperoxidase and Activated Macrophages on Metaphase II Mouse Oocyte Quality. PLoS One. 2016; 11: e0151160.

25. Grant CM. Role of the glutathione/glutaredoxin and thioredoxin systems in yeast growth and response to stress conditions. Mol Microbiol. 2001; 39: 533-41.

26. Pastore A, Federici G, Bertini E and Piemonte F. Analysis of glutathione: implication in redox and detoxification. Clin Chim Acta. 2003; 333: 19-39.

27. Jalaludeen AM, Ha WT, Lee R, et al. Biochanin A Ameliorates ArsenicInduced Hepato- and Hematotoxicity in Rats. Molecules. 2016; 21: 69. 


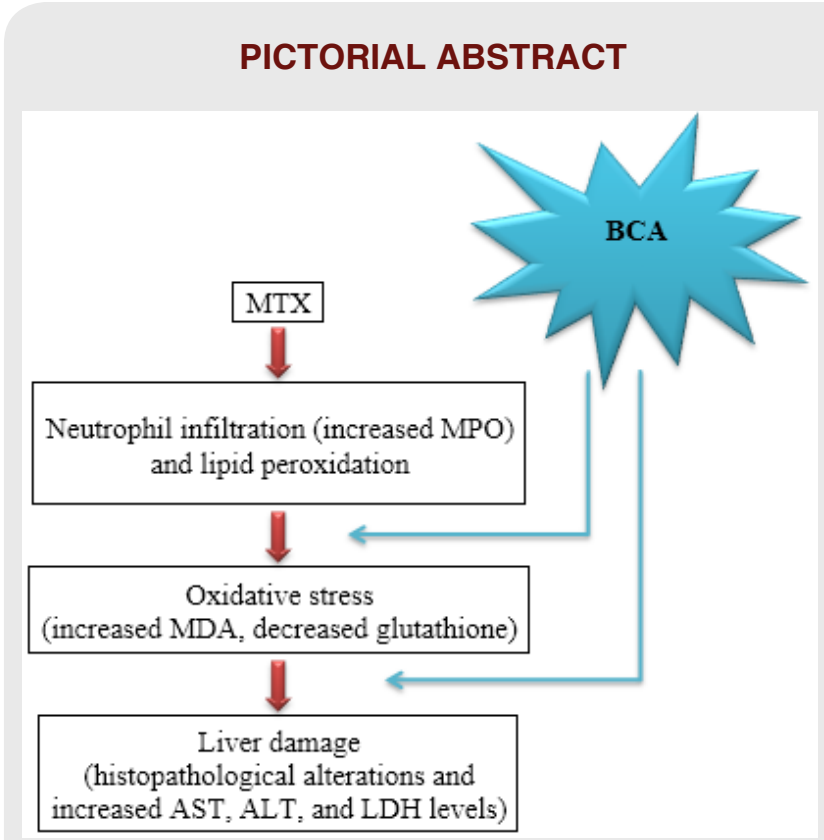

\section{SUMMARY}

- In this study, we planned to investigate the possible protective effects of BCA on MTXinduced liver injury in rats.

- Rats were injected with a single dose of MTX $(20 \mathrm{mg} / \mathrm{kg}$ ) to induce acute liver injury.

- Rats were pretreated with BCA for 5 days $(50 \mathrm{mg} / \mathrm{kg}$ ) to prevent the MTX-induced organ damage in the liver.

- BCA administration to rats prevented histopathological and biochemical alterations induced by MTX.

\section{About Authors}

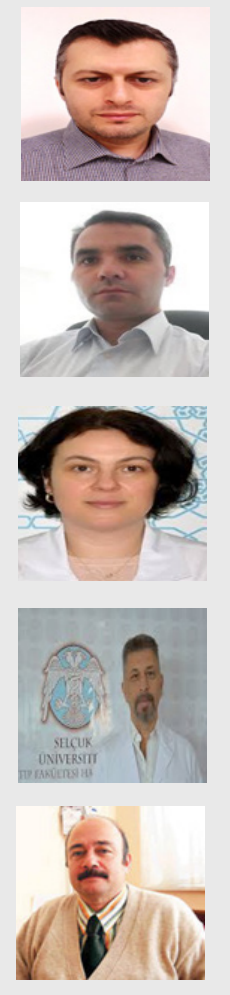

Erdem K. Ozer: Physician-specialist in Pharmacology at Faculty of Medicine, Selcuk University, Konya, Turkey.

Mustafa T. Goktas: His main research areas are pharmacogenetics and drug toxicity.

Aysun Toker: She is an Associate Professor in Biochemistry.

Ceyhan Ugurluoglu: He is an Assistant Professor in Pathology.

Hulagu Bariskaner: Professor of Pharmacology at Faculty of Medicine, Selcuk University, Konya, Turkey.

Cite this article: Ozer EK, Goktas MT, Toker A, Ugurluoglu C, Bariskaner H. Protective Effects of Biochanin A against Methotrexate-Induced Acute Liver Injury in Rats. Indian J of Pharmaceutical Education and Research. 2017;51(4S):S741-S6. 